\title{
Body impedance analyzer and anthropometric indicators; predictors of metabolic syndrome
}

\author{
Hamidreza Pouragha ${ }^{1}\left(\mathbb{D} \cdot\right.$ Mehdi Amiri $^{2} \cdot$ Maryam Saraei $^{3} \cdot$ Gholamreza Pouryaghoub $^{1}\left(\operatorname{Ramin}\right.$ Mehrdad $^{1}(\mathbb{C}$
}

Received: 7 March 2021 / Accepted: 16 June 2021 / Published online: 13 July 2021

(c) Springer Nature Switzerland AG 2021

\begin{abstract}
Aim Metabolic syndrome is one of the outcomes of a sedentary lifestyle in the modern world. In this study, we want to introduce the predictors of metabolic syndrome using anthropometric indices and Bio-Electrical Impedance Analysis (BIA) test values.

Method This cross-sectional study was performed on 2284 employees of Tehran University of Medical Sciences in different job categories. Metabolic syndrome was determined according to IDF criteria. Anthropometric dimensions, para-clinical tests, basic information were collected from the participants. Also, the body analysis of the participants was performed using a BIA method.

Result The prevalence of metabolic syndrome in this study was $23.2 \%$ based on IDF criteria, which was $21 \%$ and $26.6 \%$ in men and women, respectively. The most important factor among the components of IDF criteria was HDL deficiency. In this study, neck circumference, fat mass, visceral fat, muscle mass percentage and waist to height ratio were observed as predictors of metabolic syndrome.

Conclusion This study realized that there is association between fat mass, fat-free mass, visceral fat and muscle mass which all are some elements of body composition analysis and metabolic syndrome as a major health issue.
\end{abstract}

Keywords Metabolic syndrome $\cdot$ Body composition $\cdot$ Health personnel $\cdot$ Sedentary behavior

\section{Introduction}

Metabolic syndrome is known as a combination of diseases that increase the risk of atherosclerotic cardiovascular disease (ASCVD) and diabetes mellitus [1]. Also, with the COVID-19 disease pandemic, there is evidence of an increased risk of disease outcomes and mortality in patients

Ramin Mehrdad

mehrdadr@tums.ac.ir

Hamidreza Pouragha

pouragha_h@razi.tums.ac.ir

Gholamreza Pouryaghoub

pourya@tums.ac.ir

1 Center for Research on Occupational Diseases, Tehran University of Medical Sciences, Tehran, Iran

2 Occupational Medicine Department, School of Medicine, Tehran University of Medical Sciences, Tehran, Iran

3 Department of Occupational Medicine, School of Medicine Baharlou Hospital, Tehran University of Medical Sciences, Tehran, Iran with metabolic syndrome [2]. This risk factor consists of atherogenic dyslipidemia, elevated blood pressure, elevated plasma glucose-a pro thrombotic state, and pro-inflammatory state [1]. Metabolic syndrome doubles the risk of ASCVD and confers a 5 times higher risk of developing diabetes mellitus [1]. The International Diabetes Federation (IDF) estimates that about 25 percent of the world's population has metabolic syndrome [3].

More than one-fifth of countries in Asia and the Pacific are affected by metabolic syndrome [4]. The prevalence of metabolic syndrome in South Korea is 30.5\% and shows an increasing trend [5]. A 25-year report found that the prevalence of metabolic syndrome in all social groups in the United States was increasing, with more than one-third of American adults having metabolic syndrome [6]. In a metaanalysis, the prevalence of metabolic syndrome in Middle Eastern countries is estimated at 25\% [7]. The prevalence of metabolic syndrome in Saudi Arabia is $31.6 \%$ according to the IDF criteria [8]. About one-third of Iranian adults have metabolic syndrome [9]. In other studies, metabolic syndrome has been reported $34.2 \%$ in Iran [10] also in a 
report, the prevalence of metabolic syndrome in Iran was $38 \%$ according to IDF criteria [11].

Socioeconomic background and race are influential in metabolic syndrome [3]. In previous studies, Metabolic syndrome has been associated with cancers (breast, pancreatic, colon, and liver) [3]. In a study, the most prevalent component influencing metabolic syndrome is atherogenic dyslipidemia, which is associated with a decrease in HDL and an increase in cholesterol [12]. Also, the most important and common cause of metabolic syndrome was low HDL with a prevalence of $59.7 \%$ in Iran [13].

Obesity and atherogenic dyslipidemia (HDL deficiency and high triglycerides), high blood pressure, high blood sugar are among the main criteria of metabolic syndrome that have been introduced by various scientific societies such as the International Diabetes Federation (IDF), World Health Organization (WHO), the National Cholesterol Education Program (NCEP) Adult Treatment Panel III (ATP III). In recent years, researchers have tried to introduce other predictors of metabolic syndrome in addition to the criteria that determine metabolic syndrome. Predictors of metabolic syndrome fall into three main categories. The first category is anthropometric indicators that are introduced by measuring body dimensions and their ratios. The second category of body composition is introduced with newer technologies such as Bioelectrical Impedance Analysis (BIA) and the third category are other values such as biochemical indicators and so on. Anthropometric indices and ratios predicting metabolic syndrome can be mentioned as follows: Body Mass Index BMI [14-23]; Waist Circumference WC [15-18, 21-26]; Neck circumference [27]; Waist to Hip Ratio WHR [17, 18, 20, 21, 24, 26]; Waist to Height Ratio WHtR [15-18, 20, 21, 26, 28, 29] and Neck to Height Ratio [30]. Among the body composition variables that can be used as predictors of metabolic syndrome, the following predictors have been introduced in the previous study; body fat percentage and fat mass index [15, 20, 31]; Muscle mass [31]; Fat-free mass [15, 20]; Visceral adiposity index and Visceral fat [16, 17, 23, 28, 32-34]; sarcopenic obesity [35]; Lipid accumulation product LAP [5, 28]; Body adiposity index [23, 28]; Fat to muscle ratio [36] and Muscle to visceral fat ratio [36]. Other predictors of metabolic syndrome include Nonfunctioning adrenal incidentaloma NFAI [37], High TSH and subclinical hypothyroidism [38] and Uric acid [39].

Given the above and the high prevalence of metabolic syndrome in developed and developing countries, especially Iran, the need for a comprehensive and insightful look at the factors involved in metabolic syndrome is inevitable. In the following study, we try to examine some variables and predictors of metabolic syndrome among health personnel. According to this, we simultaneously examine anthropometric indices and ratios such as BMI and WHtR, and BIA variables among health service staff to realize which one of these indices is a predictor of metabolic syndrome.

\section{Methods}

This cross-sectional study was conducted in 2018-2019 as a measure of enrollment data in the Tehran University of Medical Sciences' Cohort (TEC) Study [40]. The participants in this study were $60.7 \%$ female, $39.3 \%$ male with a mean (SD) age of 43.7 (8.6) years. Participants in the study included a variety of departments; including office workers, clinical workers, laboratory staff, services workers, and security guard staff. These types of jobs are considered as permanent or contractual. Job information, type of job, type of employment, and work experience were also obtained from the participants.

\section{Participants}

Participants in this study were 2284 staff of Tehran University of Medical Sciences in various job categories, of which 898 were men and 1386 were women. In this study, part of the data collected in the TEC cohort enrollment phase was used. The sample size is given in the main study protocol article [40]. All participants voluntarily signed the informed consent form at the study center. All examinations and data collection were performed in one working day at the designated center by trained personnel and according to the approved protocol of the study. All data collection steps were supervised by independent quality control and assurance team. Inclusion criteria in this study were employment relationship with Tehran University of Medical Sciences. All staff were allowed to enter the study voluntarily. Also, there were no exclusion criteria.

\section{Blood samples}

All participants were asked to fast for $12 \mathrm{~h}$ before attending the study. $10 \mathrm{~mL}$ of venous blood was collected using a sodium fluoride tube from each participant (between 8 and $9 \mathrm{am}$ ). Plasma was separated by a 15 -min centrifuge at $3000 \mathrm{rpm}$. Plasma was stored at $-20^{\circ} \mathrm{C}$ at the laboratory. Levels of high-density lipoprotein (HDL), low-density lipoprotein (LDL), and triglycerides were measured, using the glycerokinase oxidase/peroxidase method. The blood glucose was determined, using the glucose oxidase/peroxidase method; and blood cholesterol was measured using the esterase oxidase/peroxidase method [41]. 


\section{Blood pressure measurement}

The blood pressure of the participants was measured three times and the average was reported with a precision of one $\mathrm{mm} \mathrm{Hg}$. We measured the blood pressure of participants in a sitting position after a 15 -min break. There was a thirtyminute interval between the first and second round measurements and a two-hour interval between the second and third rounds. Blood pressure was measured using a standard and calibrated clinical mercury manometer [42].

\section{Anthropometry}

The weight of the participants in light clothing was measured by the SECA scales with an accuracy of $0.1 \mathrm{~kg}$. Height was also measured using the SECA stadiometer to an accuracy of one millimeter. Based on height and weight data, the BMI index was calculated by dividing weight $(\mathrm{kg})$ by height squared $\left(\mathrm{m}^{2}\right)$. Also, all circumferences were measured with an accuracy of one millimeter by a standard tape soft meter. Waist circumferences were measured while subjects were standing and measured as the minimum abdominal circumference between the xiphoid process and the umbilicus. Hip circumferences were measured as the maximum circumferences over the buttocks. Also, the waist to hip ratio (WHR) circumference is the result of dividing the circumference of the abdomen around the buttocks and the waist to height ratio (WHtR) divides abdomen circumference by height [43].

\section{Body analyzer}

One of the common methods for studying and analyzing body shape is the body composition analysis method, which is available with different technologies. There are various methods for analyzing body composition, including the use of Caliper, anthropometry, tracer dilution, densitometry, air displacement plethysmography, dual-energy X-ray absorptiometry, bioelectrical impedance analyzer (BIA), computed tomography, magnetic resonance imaging, 3D body scanning. In the BIA method, the impedance of different tissues of the body is analyzed and the composition of the body is predicted. A very weak electrical current of 800 microamperes with a frequency of $50 \mathrm{kHz}$ is sent to the body and the impedance of the tissues is measured against this current. Water has a high conductivity due to the presence of electrolytes, but adipose tissue has a low conductivity [44, 45].

In the present study, body composition analysis was performed using Tanita-720 body analyzer made in Japan. At the time of measurement, all metal parts such as watches, rings, and other jewelry were removed from the person. All measurements were performed by the same trained personnel based on the same protocol. The variables studied included: Body Fat\% (Percentage of total body fat), Muscle Mass\% (Percentage of total muscle tissue in the body), Visceral Fat, Bone Mass (The mass of the bones), Fat-Free Mass FFM (Bodyweight excluding fat), Total Body Water (TBW), Extra Cellular Water (ECW), Intra Cellular Water (ICW).

\section{Physical activity}

Physical activity was calculated using the short form of the IPAQ (International Physical Activity Questionnaire) and MET (metabolic equivalent of task) hours per week (METhours/week). The MET scores for intense, medium, and hiking activities (for at least $10 \mathrm{~min}$ ) were multiplied by the time each participant spent on the activity, taking into account the frequency of participation in the activities mentioned over the past week. Then, to get the sum, the scores for the various activities were summarized as MET-min/ week. Finally, they fall into three categories: low, medium, and high activity [46].

Smoking status We divided participants based on the smoking status into three categories. People who have smoked for at least one day in the last 30 days as Current smokers or an adult who has smoked 100 cigarettes in his or her lifetime. People who used to smoke but do not smoke now (have not smoked a single cigarette in the last month), a Former smoker, or an adult who has smoked at least 100 cigarettes in his or her lifetime but who had quit smoking. And people who have never smoked in their lifetime, as Never smoker (an adult who has never smoked, or who has smoked less than 100 cigarettes in his or her lifetime) [47].

\section{Met-S and its components}

In the present study, Met-S was defined according to International Diabetes Federation (IDF) criteria. Diagnostic criteria for Met-S based on IDF criteria [42] are:

- Obesity based on abdominal circumference greater than $94 \mathrm{~cm}$ among men and greater than $80 \mathrm{~cm}$ among women or BMI above $30 \mathrm{~kg} / \mathrm{m}^{2}$.

Plus, two of the following:

- Raised blood triglyceride (TG) is more than $150 \mathrm{mg} / \mathrm{dl}$ or specific treatment for this lipid abnormality.

- Reduced HDL cholesterol levels less than $40 \mathrm{mg} / \mathrm{dl}$ among men and less than $50 \mathrm{mg} / \mathrm{dl}$ among women or specific treatment for this lipid abnormality.

- Raised blood pressure, Systolic blood pressure (SBP) greater than $130 \mathrm{mmHg}$ or diastolic blood pressure 
(DBP) greater than $85 \mathrm{mmHg}$ or treatment of previously diagnosed hypertension.

- Raised fasting plasma glucose greater than $100 \mathrm{mg} / \mathrm{dl}$ or if a person is on high blood sugar medication or previously diagnosed with type 2 diabetes.

Met-S was assessed according to the above criteria.

\section{Statistics}

To describe qualitative data, we used frequency and percentage and for quantitative data mean and standard deviation were used. We also tested all quantitative variables for normal distribution using the Kolmogorov-Smirnov test. For quantitative data with non-normal distribution, we described the variable with mean, standard deviation, median, and range.

For univariate analysis, we used the chi-square test for association between two qualitative variables. Also, for the association between qualitative and quantitative variables, independent t-test, analysis of variance, Mann-Whitney U test, Wilcoxon, and Kruskal-Wallis were used based on the type and normality of variable. We also used logistic regression analysis to realize which variables are predictors of metabolic syndrome. Data analysis was performed using the Chicago SPSS-24 software. A P-value less than 0.05 was considered as significant.

\section{Ethical considerations}

This study was done with the approval of the ethics committee of Tehran University of Medical Sciences with the ethics code IR.TUMS.VCR.REC.1395.1484, IR.TUMS. VCR.REC.1398.246. All participants participated in this study voluntarily and with informed consent, and signed the form of informed participation in the research plan approved by the ethics committee before starting the data gathering process. Participants were free to withdraw from the study at any stage. All the identity information of the participants was registered in complete confidentiality and by the study protocol in the form of a code that was provided to the researchers to analyze the information without name and mark, or any information revealing the identity of the individuals. The authors confirm that all steps and methods have been performed according to the approval of the ethics committee of Tehran University of Medical Sciences. This study was carried out according to the Helsinki Declaration Principles.

\section{Result:}

This study was performed on 2284 employees of Tehran University of Medical Sciences. $60.7 \%$ were female and $39.3 \%$ were male. The mean (SD) age of the participants was 43.7(8.6) years, the mean (SD) age of women and men was 43.1(8.3) and 44.6(9.0) respectively. Participants were divided into seven occupational groups; office workers $(51.4 \%)$, healthcare workers $(7.5 \%)$, services workers $(7.7 \%)$, technical staff $(2.7 \%)$, laboratory staff $(4.2 \%)$ security $(3.4 \%)$ and other $(3.1 \%)$. The participant's height mean (SD) was 165.1(9.0), which was 159.7(5.6) and 173.4(6.7) for women and men respectively. The mean (SD) of participants' weight was 73.5(14.5) which was 67.2(11.3) for women and 83.1 (13.6) for men.

Also, the mean (SD) of the participants' BMI was 26.9(4.3), which was 26.4(4.4) for women and 27.6 (4.1) for men. In the BMI classification, $1.1 \%$ of the participants were underweight $\left(\mathrm{BMI}<18.5 \mathrm{~kg} / \mathrm{m}^{2}\right), 35.1 \%$ were in the normal range $\left(18.5-24.9 \mathrm{~kg} / \mathrm{m}^{2}\right), 42.7 \%$ were overweight $\left(25-29.9 \mathrm{~kg} / \mathrm{m}^{2}\right)$ and $21.1 \%$ were obese (BMI $\left.\geq 30\right)$. Among all participants, $17.1 \%$ had class 1 obesity $\left(30-34.9 \mathrm{~kg} / \mathrm{m}^{2}\right), 3 \%$ had class 2 obesity $\left(35-39.9 \mathrm{~kg} / \mathrm{m}^{2}\right)$, and $1 \%$ had class 3 obesity (BMI $\geq 40)$.

The prevalence of metabolic syndrome according to IDF criteria in this study was $23.2 \%$. Prevalence of metabolic syndrome in men and women was $21 \%$ and $26.6 \%$, respectively. According to Table 1, other measured anthropometric values such as waist circumference, neck circumference, and also the ratio of waist circumference to height have been reported. Accordingly, in this study, among anthropometric variables, the only difference was in height among participants with metabolic syndrome, and for other anthropometric variables, the difference was significant, $\mathrm{p}<0.05$.

FBS, TG, and HDL have also been reported as biochemical variables as criteria for metabolic syndrome. The highest prevalence of these variables in this study is related to the HDL. That is considered as the main cause of metabolic syndrome among the main components of metabolic syndrome. Accordingly, HDL levels less than $40 \mathrm{mg} / \mathrm{dl}$ in men and less than $50 \mathrm{mg} / \mathrm{dl}$ in women have a prevalence of $54.4 \%$. The prevalence of low level HDL was $42.7 \%$ in men and $62 \%$ in women. The prevalence of hypertension among participants in this study according to IDF definition was $23.8 \%$. The prevalence of hypertension was $18.7 \%$ in women and $31.5 \%$ in men, respectively.

The values and variables obtained from the analysis of body composition of participants include: Fat mass, body fat percentage, fat-free mass, visceral fat, muscle mass, bone mass, TBW, ICW, ECW, and the fat to muscle ratio, the difference between patients with metabolic syndrome and healthy was significant, $\mathrm{p}<0.05$. 


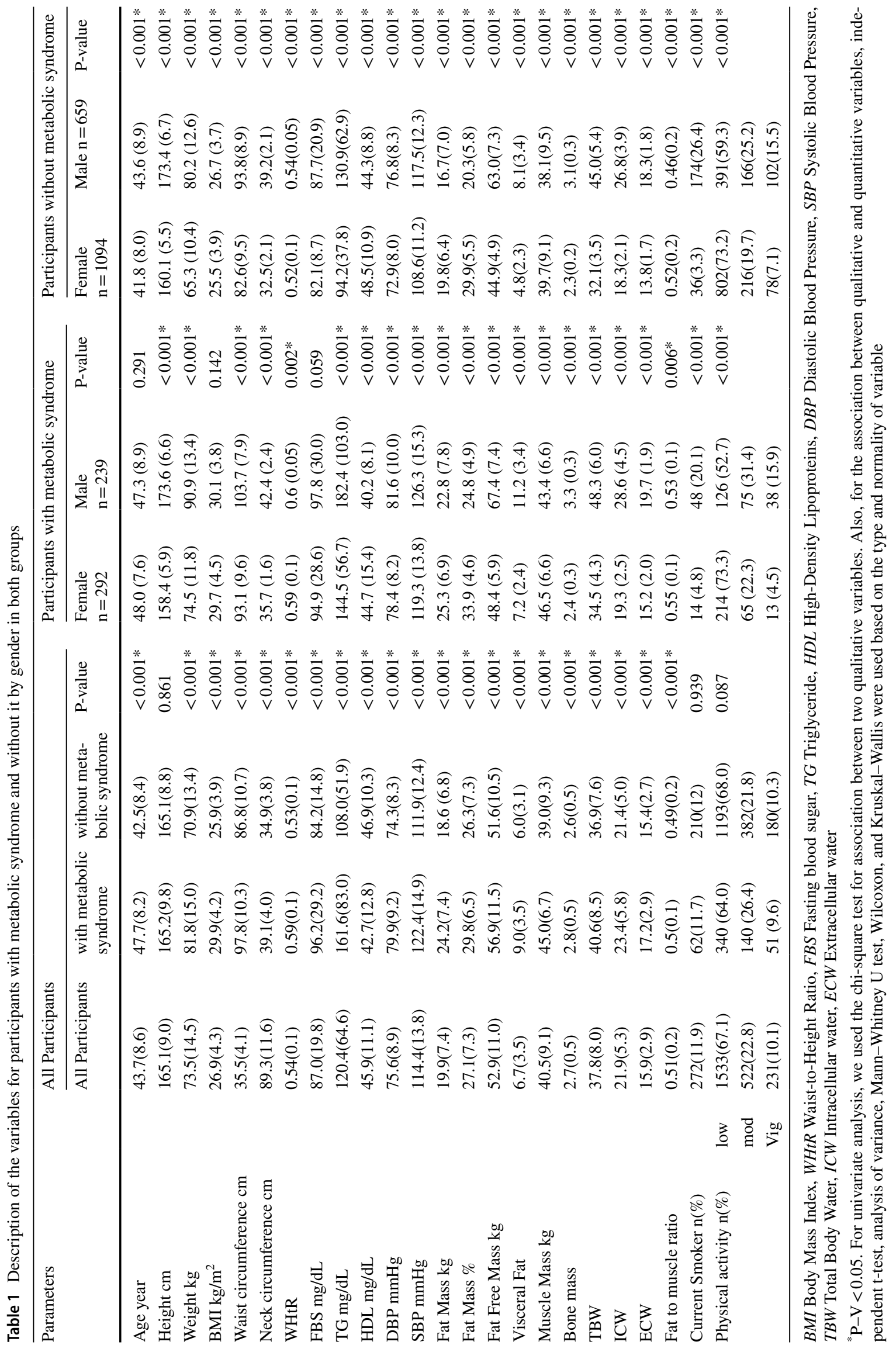


$11.7 \%$ of study participants were current smoker and the difference between smoking in participant with metabolic syndrome and participants without metabolic syndrome was not significant, $p=0.939$. In addition, the physical activity of participant with metabolic syndrome and without metabolic syndrome group was not statistically significant, $\mathrm{p}>0.05$.

None of the participants in the underweight range below $18.5 \mathrm{~kg} / \mathrm{m}^{2}$ had metabolic syndrome. The prevalence of metabolic syndrome among normal BMI participants $\left(18.5-24.9 \mathrm{~kg} / \mathrm{m}^{2}\right)$ was $5.4 \%$. The prevalence of metabolic syndrome in overweight individuals (with a BMI range of 25-29.9) was $25.9 \%$. Also, the prevalence of metabolic syndrome in class I obese people (BMI $30-34.9 \mathrm{~kg} / \mathrm{m}^{2}$ ) was $47.6 \%$. The prevalence in people with class II obesity (BMI between 35 and $39.9 \mathrm{~kg} / \mathrm{m}^{2}$ ) was $47.8 \%$ and the prevalence of metabolic syndrome in people with class III obesity and $\mathrm{BMI} \geq 40 \mathrm{~kg} / \mathrm{m}^{2}$ was $69.6 \%$. These results suggest that obesity alone can significantly increase the risk of metabolic syndrome. The prevalence of metabolic syndrome in different groups in terms of BMI classification was significant, $\mathrm{P}<0.05$.

Table 1 shows all the variables by gender for participants with metabolic syndrome and without metabolic syndrome group. All participants in this study were classified into seven occupational groups. The highest prevalence of metabolic syndrome was observed in the group of security staff with a prevalence of $37.7 \%$. Prevalence of metabolic syndrome among other groups were: office workers $23.6 \%$, healthcare workers $21 \%$, service personnel $27.7 \%$, technical staff $29 \%$, laboratory personnel $15.8 \%$, and other personnel $15.3 \%$. The difference between prevalence of metabolic syndrome for different occupational groups was statistically significant, $\mathrm{p}<0.05$.

To determine the predictors of metabolic syndrome other than the main components of metabolic syndrome based on IDF criteria, we used binary logistic regression. Neck circumference, fat mass, fat free mass, visceral fat and fat percentage and waist-to-height ratio were independently observed as predictors of metabolic syndrome adjusted for sex and age with R square $=0.541$ (Table 2 ).

\section{Discussion}

Many studies on the factors affecting metabolic syndrome have been performed, also previous studies have attempted to introduce predictors of metabolic syndrome. Predictors of metabolic syndrome can be classified into three categories: anthropometric variables, biochemical variables, and lifestyle.

In the present study, the association of body composition variables and metabolic syndrome were studied in the employees of a large university with the objective of providing health, medical and educational services with a staff of over 20,000 people. For this purpose, 2286 university staffs were examined and their basic variables, body analysis, anthropometry, and lifestyle on metabolic syndrome were examined.

The results of this study showed that participants with metabolic syndrome are older than healthy participants. There was no significant difference of age between men and women with metabolic syndrome. According to the findings of this study, metabolic syndrome occurs in both sexes in almost the same age range. This finding has been widely observed in previous studies [48-50]. Probably the difference in prevalence of metabolic syndrome between genders can be attributed to elements other than age. Differences in the prevalence of metabolic syndrome among women and men have also been observed in previous studies [51-56].

There was no significant difference of height between participants with metabolic syndrome and healthy participants. Of course, in terms of gender, the difference between the height of men and women in both groups with and without metabolic syndrom was significant.

Participants with metabolic syndrome were heavier in both sexes than healthy participants. This similarity was
Table 2 Logistic regression for Metabolic Syndrome Predictors

\begin{tabular}{lccccc}
\hline & & & & \multicolumn{2}{c}{$95 \%$ C.I. for Odds Ratio } \\
\cline { 5 - 6 } Variable & B & Odds Ratio & P-value & Lower & Upper \\
\hline Sex (F/M) & 1.39 & 4.03 & 0.366 & 0.20 & 82.98 \\
Age (year) & 0.08 & 1.09 & 0.063 & 1.0 & 1.19 \\
Neck circumferences & 0.05 & 1.05 & $<0.001^{*}$ & 1.03 & 1.083 \\
Fat Mass & 0.24 & 1.27 & $0.007^{*}$ & 1.07 & 1.51 \\
Fat Free Mass & -22.19 & $<0.001$ & $0.008^{*}$ & $<0.001$ & 0.003 \\
Visceral fat & -16.0 & $<0.001$ & $<0.001^{*}$ & $<0.001$ & 0.001 \\
Muscle Mass Percent & 23.19 & $1.18 \times 10^{10}$ & $0.008^{*}$ & 455.2 & $3.05 \times 10^{17}$ \\
Waist to Height Ratio & 28.92 & $3.61 \times 10^{12}$ & $0.001^{*}$ & $162,043.5$ & $8.05 \times 10^{19}$ \\
Constant & -34.90 & $<0.001$ & $<0.001^{*}$ & 0.20 & 82.98 \\
\hline
\end{tabular}

$\mathrm{R}$ Square $=0.541$ 
also observed with slight differences in BMI. In the IDF criteria, BMI is one of the primary criteria for the classification of metabolic syndrome. BMI was not significantly different between the two sexes and was almost in the same range in both groups. However, in healthy participants, the difference in BMI was significant. In the healthy group, men and women were overweight, so that the average BMI of healthy participant's women and men was 25.5 and 26.7 respectively.

One of the anthropometric variables that was not a criterion for the inclusion of metabolic syndrome is neck circumference. In this study, it was observed that neck circumference is significantly different between participants with and without metabolic syndrome. In the group of metabolic syndrome, this difference is due to gender because there is a significant difference in the healthy group. There was also a significant difference in neck circumference between metabolic syndrome women and healthy women. This difference was also observed in the comparison between metabolic syndrome and healthy men. Previous studies have reported such an association [57-70].

There was a significant difference between waist to height ratio in participants with metabolic syndrome and healthy participants. This difference was also significant in comparing women with and without metabolic syndrome. This significant difference was also observed between men. These observations were mentioned in other studies [71-78].

Fasting Blood Sugar is one of the criteria for metabolic syndrome, there was a significant difference between participants with and without metabolic syndrome. But there was no difference in terms of gender, i.e., it was almost equally high in both groups of men and women.

There was a significant difference between triglyceride and HDL levels in both metabolic syndrome and healthy groups, but in participants with metabolic syndrome, TG level was higher in men than women and HDL level in women was significantly higher than men. This difference was also observed in healthy participants.

Systolic and diastolic blood pressure were significantly different in both metabolic syndrome and healthy groups. Among the metabolic syndrome group, men had higher blood pressure than women. This difference between the two genders was also observed in the healthy group.

The variables of Bio-Electrical Impedance Analysis to the determination of body composition that were studied in this study included fat mass, fat-free mass, visceral fat, muscle mass, bone mass, total body water, extracellular water (ECW), intracellular water (ICW), and also the fat to muscle ratio. In all these variables, there was a significant difference between participants with metabolic syndrome and without metabolic syndrome. Also, there was an association between women and men with and without metabolic syndrome.
Smoking did not show association with metabolic syndrom, but there was a significant difference between the two sexes so that men smoked more than women. Physical activity did not show an association between participants with metabolic syndrome and healthy ones, and a difference was observed between men and women.

BIA and anthropometric variables in addition to the constituent components of metabolic syndrome which were significant in univariate analysis entered to logistic regression, age and sex were not independent predictors of metabolic syndrome. The variables of neck circumference, fat mass, fat-free mass, visceral fat, muscle percentage, and waist-toheight ratio are independent predictors of metabolic syndrome adjusted for sex and age. In one study, BMI, waist circumference, waist-to-height ratio, and VAI variables were introduced as useful variables to assess the cardio-metabolic risk factors [16].

Based on the present study, waist to height ratio and visceral fat were introduced as predictors of metabolic syndrome. In other studies, abdominal circumference and body mass index were also introduced as predictors of metabolic syndrome [14, 24]. Body mass index and abdominal circumference, which are the main criteria of metabolic syndrome, were not included in this model.

The IDF and other similar criteria such as NTP including waist circumference and body mass index are defined as metabolic syndrome. Therefore, in the present study, these variables were not included in the regression model as predictors of metabolic syndrome, and we intended to examine other variables in addition to the main components of the criterion as being associated with metabolic syndrome. Neck circumference is also known as a predictor of metabolic syndrome. Neck circumference in another study in the Chinese population showed similar results [27]. Another study found that the ratio of abdominal circumference to pelvic circumference was a better predictor of the ratio of abdominal circumference to height in men, they found that the waist to hip ratio was a better predictor of the WHtR in men [18]. In the present study, the WHtR was observed as a predictor of metabolic syndrome in both sexes.

Also in the present study, fat mass, fat-free mass, visceral fat and muscle mass were introduced as predictors of metabolic syndrome along with anthropometric variables. Based on the observations of the present study, the elements obtained from the analysis of body composition by the BIA method are considered as predictors of metabolic syndrome. One of the findings observed in this study was an association between Fat-free mass and metabolic syndrome. On the other hand, the percentage of muscle, total fat mass and visceral fat are also associated with metabolic syndrome and are considered as predictors of this syndrome. As can be seen, all the main structures of body composition analysis are associated with metabolic syndrome. 
From the observations of this study, it can be concluded that body composition analysis variables can be used as an early aid in the preventive diagnosis of metabolic syndrome, considering that the results of logistic regression analysis have been adjusted for age and sex. The use of body composition analysis by BIA variables along with anthropometric measures can be a useful tool for predicting metabolic syndrome in adults.

one of the benefits of this study is the investigation on health personnel who have not enough physical activity. An Iranian proverb says that a potter drinks water from a broken jar! This means that experts use their expertise less for themselves and more to others.Now, this matter was considered to some extent in the present study and the health personnel were examined. The present study showed that metabolic syndrome can be predicted by a simple anthropometric method and body composition analyzer (Bio-Electrical Impedance Analysis). These predictors, which were introduced in this study, could be a prognosis for metabolic syndrome even before it occurs. Even in future studies, the risk of metabolic syndrome can be assessed and predicted according to the dimensions and predictors introduced in this study. For example, what percentage of visceral fat can increase the risk of metabolic syndrome? However, the methods proposed in this study, including anthropometry and body composition as available and inexpensive methods can help predict the metabolic syndrome.

\section{Conclusion}

This study realized that there is association between fat mass, fat-free mass, visceral fat and muscle mass which all are some elements of body composition analysis and metabolic syndrome as a major health issue.

Acknowledgements This study was supported and it was carried out by the cooperation of Tehran University of Medical Sciences employee's cohort (TEC) study.

\section{Declarations}

Conflict of interest On behalf of all authors, the corresponding author states that there is no conflict of interest.

\section{References:}

1. Bonora E, DeFronzo RA. Complications, comorbidities and related disorders.

2. Bansal R, Gubbi S, Muniyappa R. Metabolic syndrome and COVID 19: endocrine-immune-vascular interactions shapes clinical course. Endocrinology. 2020;161(10):bqaa112.
3. O’Neill S, O’Driscoll L. Metabolic syndrome: a closer look at the growing epidemic and its associated pathologies. Obes Rev. 2015;16(1):1-12.

4. Ranasinghe P, Mathangasinghe Y, Jayawardena R, Hills A, Misra A. Prevalence and trends of metabolic syndrome among adults in the asia-pacific region: a systematic review. BMC Public Health. 2017;17(1):1-9.

5. Lee SE, Han K, Kang YM, Kim S-O, Cho YK, Ko KS, et al. Trends in the prevalence of metabolic syndrome and its components in South Korea: Findings from the Korean National Health Insurance Service Database (2009-2013). PloS one. 2018;13(3):e0194490.

6. Moore J, Chaudhary N, Akinyemiju T. Metabolic syndrome prevalence by race/ethnicity and sex in the United States, National Health and Nutrition Examination Survey, 1988-2012. Rev Chronic Dis. 2017;14:E24.

7. Ansarimoghaddam A, Adineh HA, Zareban I, Iranpour S, HosseinZadeh A, Kh F. Prevalence of metabolic syndrome in Middle-East countries: meta-analysis of cross-sectional studies. Diabetes Metab Syndr. 2018;12(2):195-201.

8. Al-Rubeaan K, Bawazeer N, Al Farsi Y, Youssef AM, Al-Yahya AA, AlQumaidi H, et al. Prevalence of metabolic syndrome in Saudi Arabia-a cross sectional study. BMC Endocr Disord. 2018;18(1):1-9.

9. Farmanfarma KK, Kaykhaei MA, Adineh HA, Mohammadi M, Dabiri S, Ansari-Moghaddam A. Prevalence of metabolic syndrome in Iran: a meta-analysis of 69 studies. Diabetes Metab Syndr. 2019;13(1):792-9.

10. Salari N, Doulatyari PK, Daneshkhah A, Vaisi-Raygani A, Jalali $\mathrm{R}$, Kord Jamshidi $\mathrm{P}$, et al. The prevalence of metabolic syndrome in cardiovascular patients in Iran: a systematic review and meta-analysis. Diabetol Metab Syndr. 2020;12(1):1-10.

11. Dalvand S, Niksima SH, Meshkani R, Gheshlagh RG, SadeghNejadi S, Kooti W, et al. Prevalence of metabolic syndrome among Iranian population: a systematic review and meta-analysis. Iran J Public Health. 2017;46(4):456.

12. Nolan PB, Carrick-Ranson G, Stinear JW, Reading SA, Dalleck LC. Prevalence of metabolic syndrome and metabolic syndrome components in young adults: a pooled analysis. Prev Med Rep. 2017;7:211-5.

13. Mazloomzadeh S, Khazaghi ZR, Mousavinasab N. The prevalence of metabolic syndrome in Iran: a systematic review and meta-analysis. Iran J Public Health. 2018;47(4):473.

14. Pandey U, Midha T, Rao YK, Katiyar P, Wal P, Kaur S, et al. Anthropometric indicators as predictor of pre-diabetes in Indian adolescents. Indian Heart J. 2017;69(4):474-9.

15. Mooney SJ, Baecker A, Rundle AG. Comparison of anthropometric and body composition measures as predictors of components of the metabolic syndrome in a clinical setting. Obes Res Clin Pract. 2013;7(1):e55-66.

16. Czeczelewski M, Czeczelewski J, Czeczelewska E, GalczakKondraciuk A. Association of body composition indexes with cardio-metabolic risk factors. Obes Med. 2020;17:100171.

17. Amato M, Guarnotta V, Giordano C. Body composition assessment for the definition of cardiometabolic risk. J Endocrinol Invest. 2013;36(7):537-43.

18. Esmaillzadeh A, Mirmiran P, Azizi F. Waist-to-hip ratio is a better screening measure for cardiovascular risk factors than other anthropometric indicators in Tehranian adult men. Int $\mathbf{J}$ Obes. 2004;28(10):1325-32.

19. Unno M, Furusyo N, Mukae H, Koga T, Eiraku K, Hayashi J. The utility of visceral fat level by bioelectrical impedance analysis in the screening of metabolic syndrome-the results of the Kyushu and Okinawa Population Study (KOPS). J Atheroscler Thromb. 2012;19:462-70. 
20. Xu T, Liu J, Liu J, Zhu G, Han S. Relation between metabolic syndrome and body compositions among Chinese adolescents and adults from a large-scale population survey. BMC Public Health. 2017;17(1):1-8.

21. Pasdar Y, Moradi S, Moludi J, Saiedi S, Moradinazar M, Hamzeh $\mathrm{B}$, et al. Waist-to-height ratio is a better discriminator of cardiovascular disease than other anthropometric indicators in Kurdish adults. Sci Rep. 2020;10(1):1-10.

22. Deshmukh P, Gupta S, Dongre A, Bharambe M, Maliye C, Kaur S, et al. Relationship of anthropometric indicators with blood pressure levels in rural Wardha. Indian J Med Res. 2006;123(5):657.

23. de Oliveira CM, Ulbrich AZ, Neves FS, Dias FAL, Horimoto ARVR, Krieger JE, et al. Association between anthropometric indicators of adiposity and hypertension in a Brazilian population: Baependi Heart Study. PLoS One. 2017;12(10):e0185225.

24. Perona JS, Schmidt-RioValle J, Rueda-Medina B, Correa-Rodríguez M, González-Jiménez E. Waist circumference shows the highest predictive value for metabolic syndrome, and waist-tohip ratio for its components, in Spanish adolescents. Nutr Res. 2017:45:38-45.

25. Shen W, Punyanitya M, Chen J, Gallagher D, Albu J, Pi-Sunyer $\mathrm{X}$, et al. Waist circumference correlates with metabolic syndrome indicators better than percentage fat. Obesity. 2006;14(4):727-36.

26. Schneider HJ, Glaesmer H, Klotsche J, Bohler S, Lehnert H, Zeiher AM, et al. Accuracy of anthropometric indicators of obesity to predict cardiovascular risk. J Clin Endocrinol Metab. 2007;92(2):589-94.

27. Cui T, Yan Bh, Liu Z, Yang H, Gyan M, Ma Yx. Neck circumference: a valuable anthropometric measurement to detect metabolic syndrome among different age groups in China. Diabetes Metab Res Rev. 2018;34(3):e2966.

28. Guo S-x, Zhang X-h, Zhang J-y, He J, Yan Y-z, Ma J-1, et al. Visceral adiposity and anthropometric indicators as screening tools of metabolic syndrome among low income rural adults in Xinjiang. Sci Rep. 2016;6(1):1-8.

29. Suliga E, Ciesla E, Głuszek-Osuch M, Rogula T, Głuszek S, Kozieł D. The usefulness of anthropometric indices to identify the risk of metabolic syndrome. Nutrients. 2019;11(11):2598.

30. Selvan C, Dutta D, Thukral A, Nargis T, Kumar M, Mukhopadhyay $\mathrm{S}$, et al. Neck height ratio is an important predictor of metabolic syndrome among Asian Indians. Indian J Endocrinol Metab. 2016;20(6):831.

31. Pigłowska M, Kostka T, Drygas W, Jegier A, Leszczyńska $\mathrm{J}$, Bill-Bielecka M, et al. Body composition, nutritional status, and endothelial function in physically active men without metabolic syndrome-a 25 year cohort study. Lipids Health Dis. 2016;15(1):1-8.

32. Ozhan H, Alemdar R, Caglar O, Ordu S, Kaya A, Albayrak S, et al. Performance of bioelectrical impedance analysis in the diagnosis of metabolic syndrome. J Investig Med. 2012;60(3):587-91.

33. Cho D-H, Kim M-N, Joo HJ, Shim WJ, Lim D-S, Park S-M. Visceral obesity, but not central obesity, is associated with cardiac remodeling in subjects with suspected metabolic syndrome. Nutr Metab Cardiovasc Dis. 2019;29(4):360-6.

34. Omuse G, Maina D, Hoffman M, Mwangi J, Wambua C, Kagotho E, et al. Metabolic syndrome and its predictors in an urban population in Kenya: a cross sectional study. BMC Endocr Disord. 2017;17(1):1-11.

35. Lu C-W, Yang K-C, Chang H-H, Lee L-T, Chen C-Y, Huang K-C. Sarcopenic obesity is closely associated with metabolic syndrome. Obes Res Clin Pract. 2013;7(4):e301-7.

36. Ramírez-Vélez R, Carrillo HA, Correa-Bautista JE, Schmidt-RioValle J, González-Jiménez E, Correa-Rodríguez M, et al. Fat-tomuscle ratio: a new anthropometric indicator as a screening tool for metabolic syndrome in young Colombian people. Nutrients. 2018;10(8):1027.
37. Ribeiro Cavalari EM, de Paula MP, Arruda M, Carraro N, Martins A, de Souza K, et al. Nonfunctioning adrenal incidentaloma: a novel predictive factor for metabolic syndrome. Clin Endocrinol. 2018;89(5):586-95.

38. Waring AC, Rodondi N, Harrison S, Kanaya AM, Simonsick EM, Miljkovic I, et al. Thyroid function and prevalent and incident metabolic syndrome in older adults: the Health, Ageing and Body Composition Study. Clin Endocrinol. 2012;76(6):911-8.

39. Yu TY, Jee JH, Bae JC, Jin S-M, Baek J-H, Lee M-K, et al. Serum uric acid: a strong and independent predictor of metabolic syndrome after adjusting for body composition. Metabolism. 2016;65(4):432-40.

40. Nedjat S, Mehrdad R, Yunesian M, Pouragha H, Biagi V, Monazzam-Esmaeelpour MR. Prospective cohort study on the social determinants of health: Tehran University of Medical Sciences employeescohort (TEC) study protocol. BMC Public Health. 2020;20(1):1-7.

41. McPherson RA. Henry's Clinical Diagnosis and Management by Laboratory Methods: First South Asia Edition_e-Book: Elsevier India; 2017.

42. Petrie J, O'brien E, Littler W, De Swiet M. Recommendations on blood pressure measurement. Br Med J. 1986;293(6547):611.

43. Organization WH. Waist circumference and waist-hip ratio: report of a WHO expert consultation, Geneva, 8-11 December 2008; 2011.

44. Kuriyan R. Body composition techniques. Indian J Med Res. 2018;148(5):648.

45. Khalil SF, Mohktar MS, Ibrahim F. The theory and fundamentals of bioimpedance analysis in clinical status monitoring and diagnosis of diseases. Sensors. 2014;14(6):10895-928.

46. Vasheghani-Farahani A, Tahmasbi M, Asheri H, Ashraf H, Nedjat S, Kordi R. The Persian, last 7-day, long form of the International Physical Activity Questionnaire: translation and validation study. Asian J Sports Med. 2011;2(2):106.

47. Feng X, Qian Z, Zhang B, Guo E, Wang L, Liu P, et al. Number of cigarettes smoked per day, smoking index, and intracranial aneurysm rupture: a case-control study. Front Neurol. 2018;9:380.

48. Suastika K, Dwipayana P, Saraswati IMR, Kuswardhani T, Astika $\mathrm{N}$, Putrawan IB, et al. Relationship between age and metabolic disorders in the population of Bali. J Clin Gerontol Geriatr. 2011;2(2):47-52.

49. Ervin RB. Prevalence of metabolic syndrome among adults 20 years of age and over, by sex, age, race and ethnicity, and body mass index; United States, 2003-2006; 2009.

50. Hildrum B, Mykletun A, Hole T, Midthjell K, Dahl AA. Agespecific prevalence of the metabolic syndrome defined by the International Diabetes Federation and the National Cholesterol Education Program: the Norwegian HUNT 2 study. BMC Public Health. 2007;7(1):1-9.

51. Regitz-Zagrosek V, Lehmkuhl E, Weickert MO. Gender differences in the metabolic syndrome and their role for cardiovascular disease. Clin Res Cardiol. 2006;95(3):147.

52. Rochlani Y, Pothineni NV, Mehta JL. Metabolic syndrome: does it differ between women and men? Cardiovasc Drugs Ther. 2015;29(4):329-38.

53. Novak M, Björck L, Welin L, Welin C, Manhem K, Rosengren A. Gender differences in the prevalence of metabolic syndrome in 50-year-old Swedish men and women with hypertension born in 1953. J Hum Hypertens. 2013;27(1):56-61.

54. Lee S, Ko Y, Kwak C, Yim E-s. Gender differences in metabolic syndrome components among the Korean 66-year-old population with metabolic syndrome. BMC Geriatr. 2016;16(1):1-8.

55. Jiang B, Zheng Y, Chen Y, Chen Y, Li Q, Zhu C, et al. Age and gender-specific distribution of metabolic syndrome components in East China: role of hypertriglyceridemia in the SPECT-China study. Lipids Health Dis. 2018;17(1):1-11. 
56. Dallongeville J, Cottel D, Arveiler D, Tauber J-P, Bingham A, Wagner A, et al. The association of metabolic disorders with the metabolic syndrome is different in men and women. Ann Nutr Metab. 2004;48(1):43-50.

57. Luo Y, Ma X, Shen Y, Xu Y, Xiong Q, Zhang X, et al. Neck circumference as an effective measure for identifying cardiometabolic syndrome: a comparison with waist circumference. Endocrine. 2017;55(3):822-30.

58. Ozkaya I, Yardimci B, Tunckale A. Appropriate neck circumference cut-off points for metabolic syndrome in Turkish patients with type 2 diabetes. Endocrinol Diabetes Nutr. 2017;64(10):517-23.

59. Namazi N, Larijani B, Surkan P, Azadbakht L. The association of neck circumference with risk of metabolic syndrome and its components in adults: a systematic review and meta-analysis. Nutr Metab Cardiovasc Dis. 2018;28(7):657-74.

60. Ben-Noun L, Laor A. Relationship of neck circumference to cardiovascular risk factors. Obes Res. 2003;11(2):226-31.

61. Zhou J-y, Ge H, Zhu M-f, Wang L-j, Chen L, Tan Y-z, et al. Neck circumference as an independent predictive contributor to cardiometabolic syndrome. Cardiovasc Diabetol. 2013;12(1):1-7.

62. Yang G-r, Yuan S-y, Fu H-j, Wan G, Zhu L-x, Bu X-1, et al. Neck circumference positively related with central obesity, overweight, and metabolic syndrome in Chinese subjects with type 2 diabetes: Beijing Community Diabetes Study 4. Diabetes Care. 2010;33(11):2465-7.

63. Onat A, Hergenç G, Yüksel H, Can G, Ayhan E, Kaya Z, et al. Neck circumference as a measure of central obesity: associations with metabolic syndrome and obstructive sleep apnea syndrome beyond waist circumference. Clin Nutr. 2009;28(1):46-51.

64. Stabe C, Vasques ACJ, Lima MMO, Tambascia MA, Pareja JC, Yamanaka A, et al. Neck circumference as a simple tool for identifying the metabolic syndrome and insulin resistance: results from the Brazilian Metabolic Syndrome Study. Clin Endocrinol. 2013;78(6):874-81.

65. Silva CdCd, Zambon MP, Vasques ACJ, Rodrigues AMdB, Camilo DF, Antonio MÂR, et al. Neck circumference as a new anthropometric indicator for prediction of insulin resistance and components of metabolic syndrome in adolescents: Brazilian Metabolic Syndrome Study. Rev Paul Pediatr. 2014;32(2):221-9.

66. Morais AA, Morais UAB, Soares MMS, Romano MCC, Lamounier JA. Neck circumference in adolescents and cardiometabolic risk: a sistematic review. Rev Assoc Med Bras. 2018;64(1):54-62.

67. Alzeidan R, Fayed A, Hersi AS, Elmorshedy H. Performance of neck circumference to predict obesity and metabolic syndrome among adult Saudis: a cross-sectional study. BMC obesity. 2019;6(1):13.
68. Kumar NV, Ismail MH, Mahesha P, Girish M, Tripathy M. Neck circumference and cardio-metabolic syndrome. J Clin Diagn Res. 2014;8(7):MC23.

69. Ben-Noun LL, Laor A. Relationship between changes in neck circumference and cardiovascular risk factors. Exp Clin Cardiol. 2006;11(1): 14 .

70. Ahbab S, Ataoğlu HE, Tuna M, Karasulu L, Çetin F, Temiz LÜ, et al. Neck circumference, metabolic syndrome and obstructive sleep apnea syndrome; evaluation of possible linkage. Med Sci Monit. 2013;19:111.

71. Shao J, Yu L, Shen X, Li D, Wang K. Waist-to-height ratio, an optimal predictor for obesity and metabolic syndrome in Chinese adults. J Nutr Health Aging. 2010;14(9):782-5.

72. Hsieh SD, Muto T. Metabolic syndrome in Japanese men and women with special reference to the anthropometric criteria for the assessment of obesity: Proposal to use the waist-to-height ratio. Prev Med. 2006;42(2):135-9.

73. Schneider HJ, Klotsche J, Silber S, Stalla GK, Wittchen H-U. Measuring abdominal obesity: effects of height on distribution of cardiometabolic risk factors risk using waist circumference and waist-to-height ratio. Diabetes Care. 2011;34(1):e7.

74. Rodea-Montero ER, Evia-Viscarra ML, Apolinar-Jiménez E. Waist-to-height ratio is a better anthropometric index than waist circumference and BMI in predicting metabolic syndrome among obese Mexican adolescents. Int J Endocrinol. 2014;2014:195407.

75. Lo K, Wong M, Khalechelvam P, Tam W. Waist-to-height ratio, body mass index and waist circumference for screening paediatric cardio-metabolic risk factors: a meta-analysis. Obes Rev. 2016;17(12):1258-75.

76. Zhou D, Yang M, Yuan Z-P, Zhang D-D, Liang L, Wang C-L, et al. Waist-to-Height Ratio: a simple, effective and practical screening tool for childhood obesity and metabolic syndrome. Prev Med. 2014;67:35-40.

77. Yang H, Xin Z, Feng J-P, Yang J-K. Waist-to-height ratio is better than body mass index and waist circumference as a screening criterion for metabolic syndrome in Han Chinese adults. Medicine. 2017;96(39):e8192.

78. Bohr AD, Laurson K, McQueen MB. A novel cutoff for the waistto-height ratio predicting metabolic syndrome in young American adults. BMC Public Health. 2016;16(1):1-9.

Publisher's note Springer Nature remains neutral with regard to jurisdictional claims in published maps and institutional affiliations. 